\title{
Andexanet Alfa for Acute Major Bleeding Associated with Factor Xa Inhibitors
}

\author{
Stuart J. Connolly, M.D., Truman J. Milling Jr., M.D., John W. Eikelboom, M.D., C. Michael \\ Gibson, M.D., John T. Curnutte, M.D., Ph.D., Alex Gold, M.D., Michele D. Bronson, Ph.D., \\ Genmin Lu, Ph.D., Pamela B. Conley, Ph.D., Peter Verhamme, M.D., Ph.D., Jeannot Schmidt, \\ M.D., Saskia Middeldorp, M.D., Alexander T. Cohen, M.D., Jan Beyer-Westendorf, M.D., \\ Pierre Albaladejo, M.D., Jose Lopez-Sendon, M.D., Shelly Goodman, Ph.D., Janet Leeds, \\ Ph.D., Brian L. Wiens, Ph.D., Deborah M. Siegal, M.D., Elena Zotova, Ph.D., Brandi Meeks, \\ B.Eng., Juliet Nakamya, Ph.D., W. Ting Lim, M.Sc., Mark Crowther, M.D., and for the \\ ANNEXA-4 Investigators ${ }^{*}$ \\ Population Health Research Institute, McMaster University, Hamilton ON, Canada (S.J.C., J.W.E., \\ D.M.S., E.Z., B.M., J.N., W.T.L., M.C.); Seton Dell Medical School Stroke Institute, Austin, TX \\ (T.J.M.); Harvard Medical School, Boston (C.M.G.); Portola Pharmaceuticals, San Francisco \\ (J.T.C., A.G., M.D.B., G.L., P.B.C., S.G., J.L., B.L.W.); University of Leuven, Leuven, Belgium \\ (P.V.); Centre Hospitalier Universitaire de Clermont-Ferrand, Clermont-Ferrand (J.S.), and \\ Grenoble-Alpes University Hospital, Grenoble (P.A.) - both in France; Academic Medical Center, \\ Amsterdam (S.M.); Guy's and St. Thomas' Hospitals, King's College London, London (A.T.C.); \\ University Hospital Carl Gustav Carus Dresden, Dresden, Germany (J.B.-W.); and Hospital \\ Universitario La Paz, Madrid (J.L.-S.)
}

\section{Abstract}

BACKGROUND—Andexanet alfa (andexanet) is a recombinant modified human factor Xa decoy protein that has been shown to reverse the inhibition of factor $\mathrm{Xa}$ in healthy volunteers.

\begin{abstract}
METHODS—In this multicenter, prospective, open-label, single-group study, we evaluated 67 patients who had acute major bleeding within 18 hours after the administration of a factor Xa inhibitor. The patients all received a bolus of andexanet followed by a 2-hour infusion of the drug. Patients were evaluated for changes in measures of anti-factor Xa activity and were assessed for clinical hemostatic efficacy during a 12-hour period. All the patients were subsequently followed for 30 days. The efficacy population of 47 patients had a baseline value for anti-factor Xa activity of at least $75 \mathrm{ng}$ per milliliter (or $\searrow 0.5 \mathrm{IU}$ per milliliter for those receiving enoxaparin) and had confirmed bleeding severity at adjudication.
\end{abstract}

RESULTS-The mean age of the patients was 77 years; most of the patients had substantial cardiovascular disease. Bleeding was predominantly gastrointestinal or intracranial. The mean $( \pm \mathrm{SD})$ time from emergency department presentation to the administration of the andexanet bolus was $4.8 \pm 1.8$ hours. After the bolus administration, the median anti- factor Xa activity decreased

\footnotetext{
Address reprint requests to Dr. Connolly at connostu@phri.ca.

A complete list of the investigators in the Andexanet Alfa, a Novel Antidote to the Anticoagulation Effects of FXA Inhibitors (ANNEXA-4) study is provided in the Supplementary Appendix, available at NEJM.org.

Disclosure forms provided by the authors are available with the full text of this article at NEJM.org.
} 
by $89 \%$ (95\% confidence interval [CI], 58 to 94 ) from baseline among patients receiving rivaroxaban and by $93 \%$ (95\% CI, 87 to 94 ) among patients receiving apixaban. These levels remained similar during the 2-hour infusion. Four hours after the end of the infusion, there was a relative decrease from baseline of $39 \%$ in the measure of anti-factor Xa activity among patients receiving rivaroxaban and of 30\% among those receiving apixaban. Twelve hours after the andexanet infusion, clinical hemostasis was adjudicated as excellent or good in 37 of 47 patients in the efficacy analysis (79\%; 95\% CI, 64 to 89). Thrombotic events occurred in 12 of 67 patients (18\%) during the 30-day follow-up.

CONCLUSIONS-On the basis of a descriptive preliminary analysis, an initial bolus and subsequent 2-hour infusion of andexanet substantially reduced anti-factor Xa activity in patients with acute major bleeding associated with factor Xa inhibitors, with effective hemostasis occurring in 79\%. (Funded by Portola Pharmaceuticals; ANNEXA-4 ClinicalTrials.gov number,

NCT02329327.)

In randomized clinical trials, factor Xa inhibitors have been shown to be safe and effective for the treatment and prevention of venous thromboembolism and for stroke prevention in patients with atrial fibrillation. ${ }^{1-4}$ However, factor Xa inhibitors have been associated with major and even fatal bleeding events. ${ }^{1-4}$ Such episodes of acute major bleeding may be difficult to treat because there is no reversal agent. Andexanet alfa (andexanet) has been designed and developed specifically to reverse the effects of both direct and indirect factor $\mathrm{Xa}$ inhibitors. It is a recombinant, modified human factor Xa decoy protein that binds factor Xa inhibitors but does not have intrinsic catalytic activity. ${ }^{5,6}$ In healthy older volunteers who were receiving either apixaban or rivaroxaban, a bolus and infusion of andexanet sharply reduced both the unbound fraction of the plasma level of factor Xa inhibitor and anti-factor Xa activity, with minimal serious adverse effects. ${ }^{7}$ The Andexanet Alfa, a Novel Antidote to the Anticoagulation Effects of FXA Inhibitors (ANNEXA-4) study was designed to evaluate the use of andexanet in patients with acute major bleeding that was potentially lifethreatening. This interim report describes the 67 patients for whom data were complete as of June 17, 2016.

\section{METHODS STUDY DESIGN}

ANNEXA-4 is an ongoing, multicenter, prospective, open-label, single-group study of andexanet in patients with acute major bleeding. Starting on April 10, 2015, patients were enrolled at 20 centers in the United States, 1 center in the United Kingdom, and 1 center in Canada. The protocol and the statistical analysis plan are available with the full text of this article at NEJM.org.

Patients were eligible to participate in the preliminary analysis if they were at least 18 years of age and were reported to have received one of four factor Xa inhibitors - apixaban, rivaroxaban, edoxaban, or enoxaparin - within the past 18 hours (at a dose of at least $1 \mathrm{mg}$ per kilogram of body weight per day for enoxaparin). Acute major bleeding was defined as one or more of the following features: potentially life-threatening acute overt bleeding with signs or symptoms of hemodynamic compromise (e.g., severe hypotension, poor skin 
perfusion, mental confusion, or low cardiac output that could not otherwise be explained); acute overt bleeding associated with a decrease in hemoglobin of at least $2 \mathrm{~g}$ per deciliter or a hemoglobin level of $8 \mathrm{~g}$ per deciliter or less if no baseline hemoglobin level was available (or an investigator's opinion that the hemoglobin level would fall to $8 \mathrm{~g}$ per deciliter or less); or acute symptomatic bleeding in a critical area or organ (e.g., retroperitoneal, intraarticular, pericardial, intracranial, or intramuscular with the compartment syndrome). All the patients provided written informed consent.

Key exclusion criteria were the scheduling of surgery within less than 12 hours after presentation (with the exception of a minimally invasive surgery or procedure); intracranial hemorrhage in a patient with a score of less than 7 on the Glasgow Coma Scale (which ranges from 15 [normal] to 3 [deep coma]) or an estimated intra-cerebral hematoma volume of more than $60 \mathrm{ml}$; expected survival of less than 1 month; the occurrence of a major thrombotic event within 2 weeks before enrollment; or receipt of one of the following agents within 7 days before screening: vitamin $\mathrm{K}$ antagonist, dabigatran, prothrombin complex concentrate, or whole blood or plasma. Details regarding the inclusion and exclusion criteria are provided in Table S1 in the Supplementary Appendix, available at NEJM.org.

\section{STUDY TREATMENTS}

Patients received an andexanet bolus during a period of 15 to 30 minutes, followed by a 2hour infusion of the drug. The following doses were used: for patients who had taken apixaban or rivaroxaban more than 7 hours before the administration of andexanet, the bolus dose was $400 \mathrm{mg}$ and the infusion dose was $480 \mathrm{mg}$. For patients who had taken enoxaparin, edoxaban, or rivaroxaban 7 hours or less before the administration of the bolus dose or at an unknown time, the bolus dose was $800 \mathrm{mg}$ and the infusion dose was $960 \mathrm{mg}$. These andexanet doses were selected because their use was associated with a rapid reversal of antifactor Xa activity of $80 \%$ or more in previous studies. The dosing efficacy was confirmed by means of pharmacokinetic modeling that took into account differences in plasma protein binding and extravascular distribution of the factor Xa inhibitors. ${ }^{5}$ The 2-hour duration of infusion was chosen to exceed the expected time to achieve a definitive hemostatic plug. ${ }^{8}$ Investigators were encouraged to restart anticoagulant therapy as clinically indicated.

\section{STUDT POPULATIONS AND OUTCOMES}

The prespecified safety population included all the patients who received andexanet, and the efficacy population included only patients in whom the baseline anti-factor Xa activity was later determined to be $75 \mathrm{ng}$ per milliliter or more (or $\searrow 0.5 \mathrm{IU}$ per milliliter for those receiving enoxaparin) and the acute major bleeding episode was later adjudicated to meet the study criteria. All analyses except those related to safety were performed in the efficacy population. The two coprimary outcomes were the percent change in the anti-factor Xa activity and the rate of excellent or good hemostatic efficacy 12 hours after the andexanet infusion. Anti-factor Xa activity was measured by means of a validated chromogenic assay of factor Xa enzymatic activity. 


\section{STUDY ASSESSMENTS}

Patients were assessed at the following times: 3 hours and 15 minutes before the administration of the andexanet bolus (baseline); at the end of bolus administration (15 to 30 minutes after initiation); at the end of the 2-hour infusion; at 4 hours, 8 hours, and 12 hours after the end of the infusion; and at 3 days and 30 days. Blood samples were obtained to measure anti-factor Xa activity and the unbound fraction of the plasma level of factor Xa inhibitor during a 12-hour period. Endogenous thrombin potential was measured at baseline, at 8 hours, at 12 hours, and on day 3. Methods for measurement of these values have been described previously. ${ }^{7}$

For patients with intracranial hemorrhage, computed tomography (CT) or magnetic resonance imaging (MRI) of the head was performed within 3 hours before the initiation of the andexanet bolus and at 1 hour, 12 hours, and 30 days after the end of the infusion. Patients with intracranial bleeding were evaluated at baseline with the use of the modified Rankin scale for global disability and handicap, with scores ranging from 0 (no symptoms or disability) to 6 (death).

All adverse events were collected throughout the 30-day study period. Thrombotic events were adjudicated according to prespecified criteria (Table S4 in the Supplementary Appendix), and deaths were classified as cardiovascular or non-cardiovascular.

\section{HEMOSTATIC EFFICACY}

The independent adjudication committee reviewed each case to determine hemostatic efficacy on the basis of predetermined criteria (Table S3 in the Supplementary Appendix). Committee members also reviewed the eligibility of each patient. The hemostatic efficacy criteria were adapted from those used in a study evaluating prothrombin complex concentrate. $^{9}$

Briefly, intracranial hemorrhage was assessed by means of serial CT or MRI scans that were reviewed by an independent core laboratory. For intracerebral hemorrhage, an increase in volume of $20 \%$ or less from baseline at both 1 hour and 12 hours after infusion was considered to be excellent hemostasis, whereas an increase in volume of $35 \%$ or less from baseline at 12 hours was considered to be good. The scoring for sub-arachnoid and subdural bleeding events was similar, except that the maximal hematoma thickness was used. Other nonvisible bleeding, which included all gastrointestinal bleeding, was evaluated on the basis of both the corrected hemoglobin level and hematocrit at 12 hours, as compared with baseline, with a decrease in both hemoglobin and hematocrit of less than $10 \%$ considered to be excellent and a decrease of $20 \%$ or less and with the administration of no more than two units of additional coagulation intervention (e.g., plasma or prothrombin complex concentrate) considered to be good. (The corrected levels for hemoglobin and the hematocrit were calculated by subtracting $1 \mathrm{~g}$ per deciliter from the hemoglobin level or $3 \%$ from the hematocrit for each unit of packed red cells that was administered.)

For visible bleeding, a cessation of bleeding was considered to indicate excellent hemostatic efficacy if it occurred within 1 hour after infusion and good hemostatic efficacy if it occurred within 4 hours and no additional coagulation intervention was required. For musculoskeletal 
bleeding, factors that included pain relief, unequivocal improvement in objective signs of bleeding, and no increase in swelling were considered to indicate excellent hemostatic efficacy if they occurred within 1 hour after infusion and good hemostatic efficacy if they occurred within 4 hours.

\section{STUDY OVERSIGHT}

The study was designed and coordinated jointly by the Population Health Research Institute (PHRI) at McMaster University and Portola Pharmaceuticals. Investigators at PHRI gathered and analyzed the data. An independent data and safety monitoring board reviewed the study data. The study was overseen by an academic steering committee whose members vouch for the accuracy and completeness of the data, wrote all the drafts of the manuscript, and made the decision to submit the final draft for publication. The steering committee members and representatives of Portola Pharmaceuticals have mutual confidentiality agreements.

\section{STATISTICAL ANALYSIS}

Data are reported as means $( \pm \mathrm{SD})$ or medians and interquartile ranges for continuous variables and frequencies for categorical variables. We computed the percent change from baseline with a two-sided nonparametric confidence interval for the median. ${ }^{10}$ The rate of effective hemostasis is presented with an exact $95 \%$ confidence interval, as calculated with the use of the binomial test. All analyses were performed with the use of SAS software, version 9.4 (SAS Institute), or S-PLUS software, version 8.2 (TIBCO Software).

\section{RESULTS}

\section{STUDY PATIENTS}

The mean age of the patients was 77 years, and all the patients had a history of thrombotic events and cardiovascular disease (Table 1). All the patients received a bolus and subsequent andexanet infusion and were followed for 30 days or until death. Of the 67 patients, 32 were receiving rivaroxaban (median daily dose, $20 \mathrm{mg}$ ), 31 were receiving apixaban (median daily dose, $5 \mathrm{mg}$ ), and 4 were receiving enoxaparin. The primary site of bleeding was gastrointestinal in 33 patients (49\%) and intracranial in 28 (42\%), with other bleeding sites in $6(9 \%)$ (Table 2). The mean time from presentation in the emergency department to the initiation of the andexanet bolus was $4.8 \pm 1.9$ hours.

Twenty patients were not included in the efficacy population, since subsequent measurements of the baseline anti-factor Xa activity were less than $75 \mathrm{ng}$ per milliliter (or $<0.5 \mathrm{IU}$ per milliliter for enoxaparin) in 17 patients and were missing in 2 patients. Adjudicators ruled that 2 of these 19 patients and 1 additional patient did not meet the criteria for acute major bleeding. The study is ongoing and will enroll patients until there are 162 patients available for the efficacy analysis, with an expected safety population of approximately 230 .

\section{PHARMACODYNAMIC OUTCOMES}

In the efficacy population, among the 26 patients who were receiving rivaroxaban, the median value for anti-factor Xa activity fell from $277.0 \mathrm{ng}$ per milliliter at baseline to 16.8 
ng per milliliter at the end of the bolus administration, a relative decrease of $89 \%$ (95\% confidence interval [CI], 58 to 94) (Fig. 1). The median value for anti-factor Xa activity was $30.6 \mathrm{ng}$ per milliliter at the end of the 2-hour infusion, a relative decrease of $86 \%$ from baseline (95\% CI, 55 to 93). Four hours later, the median value was $177.7 \mathrm{ng}$ per milliliter, a relative decrease of 39\% from baseline (95\% CI, 27 to 45).

Among the 20 patients who were receiving apixaban, the median value for anti-factor Xa activity was reduced from $149.7 \mathrm{ng}$ per milliliter at baseline to $10.3 \mathrm{ng}$ per milliliter at the end of the bolus administration, a relative decrease of 93\% (95\% CI, 87 to 94). At the end of the 2-hour infusion, the median value for anti-factor Xa activity was $12.5 \mathrm{ng}$ per milliliter, a relative decrease of $92 \%$ (95\% CI, 85 to 94). Four hours later, the median value was 103.0 ng per milliliter, a relative decrease from baseline of 30\% (95\% CI, 23 to 46); subsequently, at 8 hours and 12 hours, the value for anti-factor Xa activity remained at similar levels.

The single patient in the efficacy group who was receiving enoxaparin had a reduction in anti-factor Xa activity from $0.61 \mathrm{IU}$ per milliliter to $0.15 \mathrm{IU}$ per milliliter at the end of the bolus administration; the value was $0.19 \mathrm{IU}$ per milliliter after the 2-hour infusion and 0.46 IU per milliliter at 4 hours and decreased gradually after that measure. Details regarding the effects of andexanet on endogenous thrombin potential (thrombin generation) and on the unbound fraction of the plasma level of the two direct factor Xa inhibitors are provided in Figures S1 and S2 in the Supplementary Appendix.

\section{CLINICAL OUTCOMES}

Of the 47 patients in the efficacy population, 37 were adjudicated as having excellent or good hemostasis (79\%; 95\% CI, 64 to 89), with 31 patients adjudicated as having excellent hemostasis and 6 as having good hemostasis, 12 hours after the andexanet infusion (Fig. 2). One patient could not be evaluated because of an administrative issue. Of the 9 patients who were adjudicated as having poor or no hemostatic efficacy, 5 were receiving rivaroxaban and 4 apixaban. Of these patients, 3 had gastrointestinal bleeding, 4 intracranial bleeding, and 2 other primary bleeding sites. The rates of excellent or good efficacy were $84 \%$ for gastrointestinal bleeding and $80 \%$ for intracranial bleeding, rates that were consistent in other subgroups that were examined.

Of the $10 \%$ of patients with the highest anti- factor Xa activity at the end of the infusion, 4 had received rivaroxaban and 1 had received apixaban; all received the lower dose of andexanet. The median values for anti-factor Xa activity in these patients were $487.1 \mathrm{ng}$ per milliliter (interquartile range, 298.7 to 505.8) at baseline and $327.4 \mathrm{ng}$ per milliliter (interquartile range, 283.9 to 330.1) at the end of the infusion. All these patients were adjudicated as having excellent or good hemostasis.

The mean scores on the modified Rankin scale for patients with intracranial bleeding were $2.2 \pm 1.9$ at baseline and $2.0 \pm 2.0$ at 30 days among the survivors. Before andexanet treatment, the procoagulant treatments that had been used were platelet infusion in 1 patient and plasma infusion in another. After the administration of andexanet until 12 hours, 4 patients received plasma, tranexamic acid, or platelets. 


\section{SAFETY}

Of the 67 patients in the safety population, there were no infusion reactions and no antibodies to factors $\mathrm{Xa}$ or $\mathrm{X}$ and no neutralizing antibodies to andexanet developed. Figure 3 shows the time course of thromboembolic events and deaths. Thrombotic events occurred in 12 patients (18\%), including 1 with myocardial infarction, 5 with stroke, 7 with deep-vein thrombosis, and 1 with pulmonary embolism, with some patients having more than one event. Four patients had a thrombotic event within 3 days after andexanet treatment, and the rest occurred between 4 and 30 days. There were 10 deaths (15\%), with 6 adjudicated as cardiovascular events and 4 as non-cardiovascular events. Anticoagulation was resumed in 18 patients $(27 \%)$ within 30 days, but a therapeutic dose of anticoagulation was restarted before the event in only 1 of the 12 patients with a thrombotic event. (An additional patient had received prophylactic doses of enoxaparin before the occurrence of a deep-vein thrombosis.)

\section{DISCUSSION}

In the ANNEXA-4 study, we enrolled patients with a high burden of major cardiovascular disease who had acute major bleeding associated with the use of a factor Xa inhibitor. The site of bleeding was most often gastrointestinal or intracranial; anti-factor Xa activity was considerably elevated in most patients and, as such, was likely to be a major impediment to clinical hemostasis. The administration of an andexanet bolus and infusion resulted in rapid and substantial reversal of anti-factor Xa activity. The clinical response 12 hours after the andexanet infusion, as measured by adjudicated hemostatic efficacy with the use of predetermined criteria, was excellent or good in $79 \%$ of the patients and was consistent across a variety of subgroups. After full enrollment in the ongoing study has provided adequate statistical power, further analysis should provide details regarding the relationship between the reduction in anti-factor Xa activity and clinical hemostatic outcomes.

Andexanet was infused over a period of 2 to 2.5 hours, and there was partial return of antifactor Xa activity toward pretreatment values at 4 to 4.5 hours after the initiation of andexanet. Clinical hemostatic efficacy, as assessed for 12 hours after the end of the infusion, was excellent or good in a high percentage of patients. This finding supports the idea that prolonged reversal of factor Xa inhibition may not be necessary to achieve a good hemostatic response. We cannot determine from our study how many of the patients would have achieved hemostatic efficacy without andexanet administration. It is possible that further refinements in the administration of andexanet could lead to more complete reversal of anti-factor Xa activity and improved clinical outcomes. Baseline anti-factor Xa activity was somewhat higher for patients receiving rivaroxaban than for those receiving apixaban, which may be related to a higher median daily dose of rivaroxaban $(20 \mathrm{mg})$ than of apixaban $(5 \mathrm{mg}$ ). Rates of hemostatic efficacy were similar for patients who were receiving the two agents.

The study was performed as a single-group cohort study because a randomized trial would have had logistical and ethical challenges. Nonetheless, the study was performed with a high level of rigor. Strict eligibility criteria restricted enrollment to patients with severe bleeding. Furthermore, patients were not included in the efficacy analysis if the adjudication 
committee did not agree that the severity of bleeding met the specific inclusion criteria. In addition, patients were included in the efficacy analysis only if the baseline value for antifactor Xa activity was $75 \mathrm{ng}$ per milliliter or higher, a factor that reduced the likelihood that good or excellent hemostasis would occur without reversal of anticoagulation. This cutoff level had resulted in an inhibition in thrombin generation of 30 to $35 \%$ in phase 2 studies. ${ }^{5}$ The hemostatic efficacy outcome consisted of precise and objective criteria applied by an independent adjudication committee. For example, evaluation of intracranial hemorrhage depended on a quantitative comparison of baseline and follow-up CT or MRI scans by a core laboratory.

The results of two previous studies of anticoagulation reversal in patients with acute major bleeding were recently reported; one with idarucizumab for dabigatran reversal ${ }^{11}$ and another with prothrombin complex concentrate for warfarin reversal. ${ }^{9}$ The idarucizumab study enrolled 51 patients who had clinical features that were similar to those of the patients in our study, including a mean age of 77 years and a rate of intracranial hemorrhage of $35 \%$. The study of prothrombin complex concentrate enrolled a younger population (mean age, 69 years) with arate of intracranial hemorrhage of only $12 \%$. Although the investigators in the idarucizumab study did not perform formal assessment of hemostatic efficacy, the study of prothrombin complex concentrate used efficacy criteria that were similar to those used in our study, with reports of good or excellent efficacy in $71 \%$ of patients (95\% CI, 64 to 81 ). In the idarucizumab study, there were five deaths (12\%) during 30 days of follow-up, a rate that was similar to that in our study. In the study of prothrombin complex concentrate, the rate of death at 45 days was $6 \%$, a percentage that perhaps reflected the patients' younger age and lower rate of intracranial hemorrhage. Thrombotic events occurred in $10 \%$ of 51 patients in the idarucizumab study, in $8 \%$ of 103 patients in the study of prothrombin complex concentrate, and in $18 \%$ of 67 patients in our study. The rate of reinitiation of anticoagulation was $69 \%$ by 90 days after idarucizumab treatment and $27 \%$ by 30 days after andexanet treatment; the rate of reinitiation was not reported in the study of prothrombin complex concentrate.

In conclusion, in this preliminary report of an ongoing cohort study in patients with acute major bleeding associated with the use of factor Xa inhibitors, andexanet rapidly reversed anti-factor Xa activity and was not associated with serious side effects. Effective hemostasis was achieved 12 hours after an infusion of andexanet in $79 \%$ of the patients. Thrombotic events occurred in $18 \%$ of the patients in the safety population, and $15 \%$ of the patients died during follow-up. A controlled study would be required to assess whether the frequency of these events exceeded that expected in patients at increased risk for thrombotic events.

\section{Supplementary Material}

Refer to Web version on PubMed Central for supplementary material.

\section{Acknowledgments}

Supported by Portola Pharmaceuticals. 


\section{References}

1. The EINSTEIN Investigators. Oral rivaroxaban for symptomatic venous thromboembolism. N Engl J Med. 2010; 363:2499-510. [PubMed: 21128814]

2. Patel MR, Mahaffey KW, Garg J, et al. Rivaroxaban versus warfarin in nonvalvular atrial fibrillation. N Engl J Med. 2011; 365:883-91. [PubMed: 21830957]

3. Granger CB, Alexander JH, McMurray JJV, et al. Apixaban versus warfarin in patients with atrial fibrillation. N Engl J Med. 2011; 365:981-92. [PubMed: 21870978]

4. Agnelli G, Buller HR, Cohen A, et al. Oral apixaban for the treatment of acute venous thromboembolism. N Engl J Med. 2013; 369:799-808. [PubMed: 23808982]

5. Lu G, DeGuzman FR, Hollenbach SJ, et al. A specific antidote for reversal of anticoagulation by direct and indirect inhibitors of coagulation factor Xa. Nat Med. 2013; 19:446-51. [PubMed: 23455714]

6. Ghadimi K, Dombrowski KE, Levy JH, Welsby IJ. Andexanet alfa for the reversal of Factor Xa inhibitor related anticoagulation. Expert Rev Hematol. 2016; 9:115-22. [PubMed: 26686866]

7. Siegal DM, Curnutte JT, Connolly SJ, et al. Andexanet alfa for the reversal of factor Xa inhibitor activity. N Engl J Med. 2015; 373:2413-24. [PubMed: 26559317]

8. Colman, RW., Clowes, AW., George, JN., et al. Overview of hemostasis. In: Colman, RW.Clowes, AW.George, JN., et al., editors. Hemostasis and thrombosis: basic principles and clinical practice. 5th. Philadelphia: Lippincott Williams \& Wilkins; 2006. p. 1-16.

9. Sarode R, Milling TJ Jr, Refaai MA, et al. Efficacy and safety of a 4-factor prothrombin complex concentrate in patients on vitamin $\mathrm{K}$ antagonists presenting with major bleeding: a randomized, plasma-controlled, phase IIIb study. Circulation. 2013; 128:1234-43. [PubMed: 23935011]

10. Hahn, GJ., Meeker, WQ. Statistical intervals: a guide for practitioners. New York: John Wiley; 1991.

11. Pollack CV Jr, Reilly PA, Eikelboom J, et al. Idarucizumab for dabigatran reversal. N Engl J Med. 2015; 373:511-20. [PubMed: 26095746] 


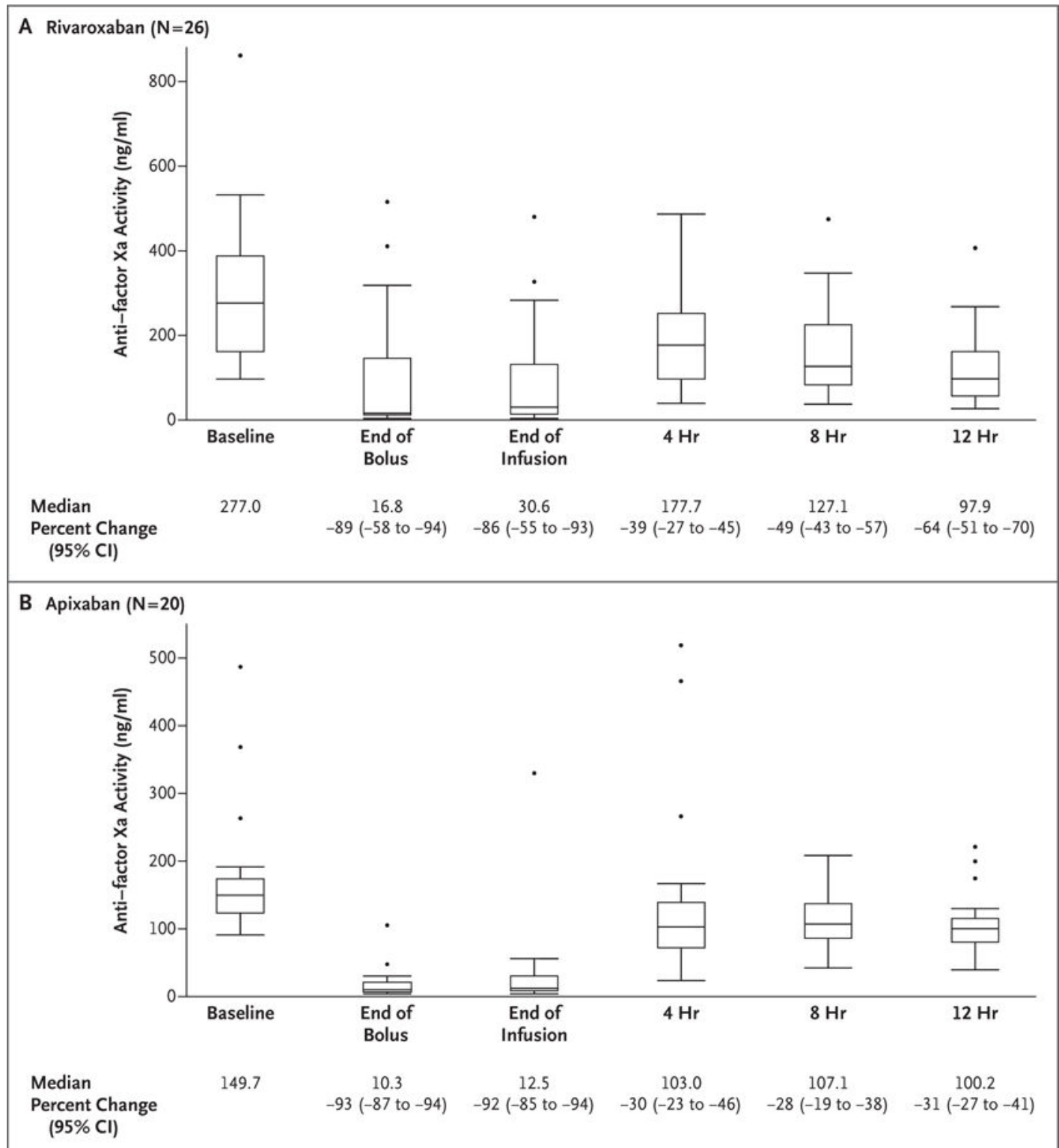

Figure 1. Anti-Factor Xa Activity and Percent Change from Baseline in Patients Receiving Rivaroxaban and Apixaban (Efficacy Population)

Shown are the values for anti-factor Xa activity (as measured by means of a validated chromogenic assay of factor Xa enzymatic activity) and the percent change from baseline in patients with acute major bleeding who received andexanet to counteract the anticoagulant effects of rivaroxaban or apixaban. Tukey box-plot values are expressed as the median (horizontal line in each box) and 25th and 75th percentiles (top and bottom of each box), with whiskers (top and bottom of each I bar) drawn to the highest data point within 1.5 times the upper quartile and to the lowest data point within 1.5 times the lower quartile. Outliers are shown as dots. The bolus of andexanet was administered during a period of 15 to 30 minutes, and the drug infusion lasted 2 hours. Subsequent time points are measured from the end of the infusion. The scales of the y axes for the two plots differ to allow proper demonstration of outliers. One patient in the efficacy population was receiving enoxaparin 
and is not included in these plots. The numbers below the graphs show the median values for anti-factor Xa activity, the percent change in median values from baseline, and 95\% confidence intervals (CI) for this change. 


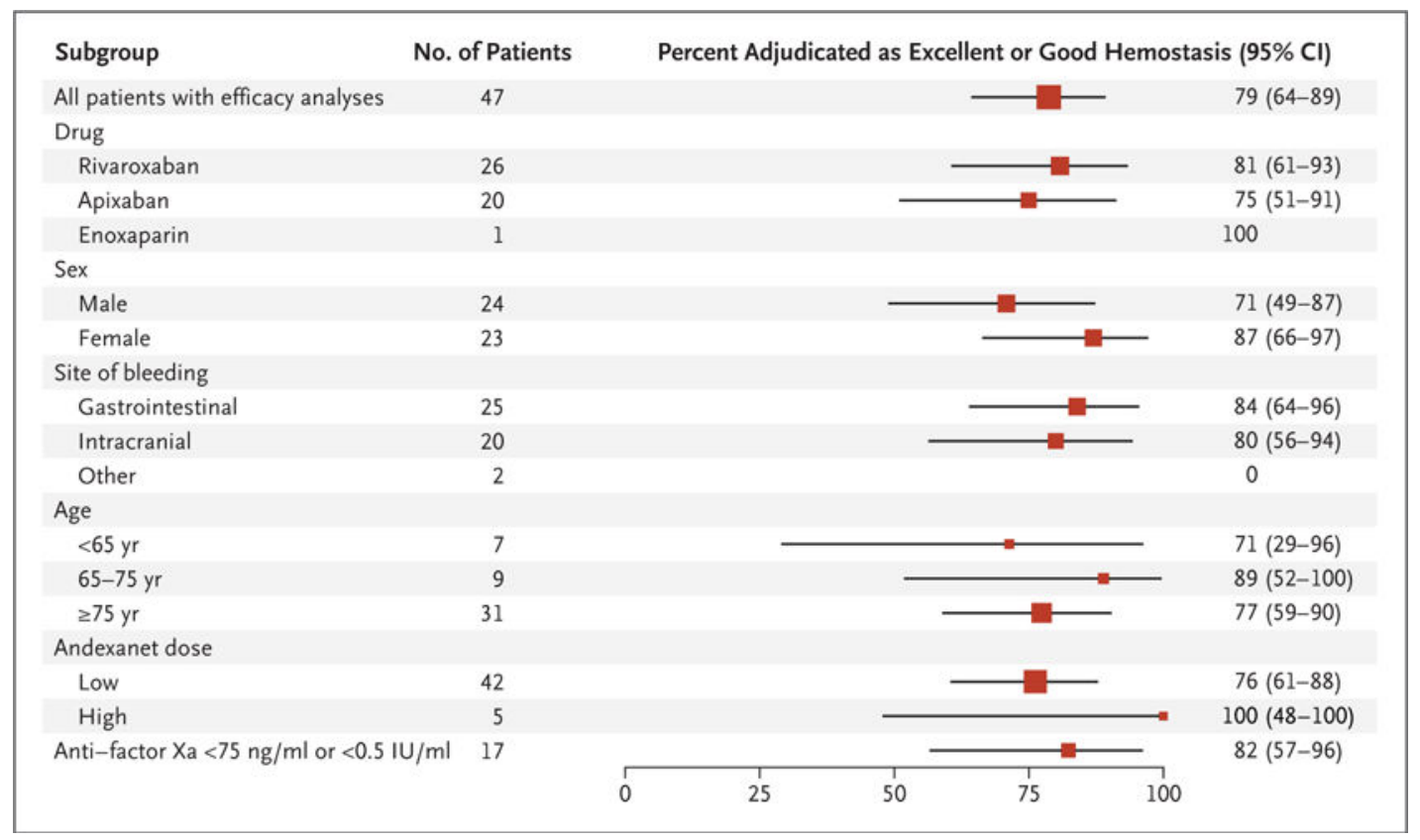

Figure 2. Subgroup Analysis of Hemostatic Efficacy

Shown are the percentages of patients in the efficacy population who were adjudicated as having excellent or good hemostatic efficacy 12 hours after treatment with andexanet on the basis of prespecified criteria (Table S3 in the Supplementary Appendix). The size of the data points is proportional to the number of patients included in the subgroup analysis. 


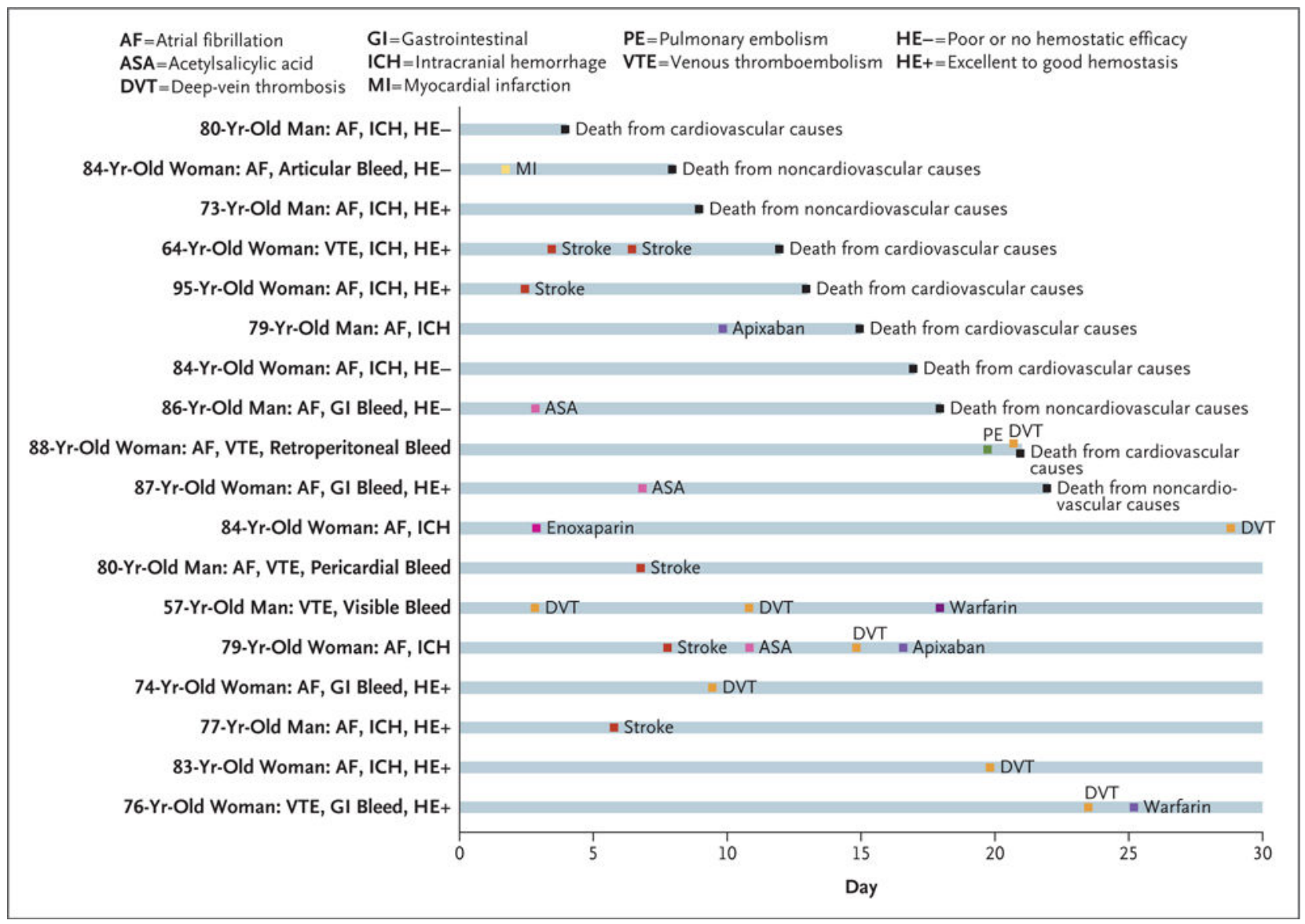

Figure 3. Thrombotic Events or Death during the 30-Day Study Period

Shown are timeline data for 18 of 67 patients in the safety population who had a thrombotic event or died after being treated with andexanet, starting with the bolus administration as baseline. Included are the patient's age and sex, the indication for anticoagulation, and the site of the acute major bleeding event. The result of adjudication of hemostatic efficacy for patients who were included in the efficacy analysis is shown as excellent or good hemostasis (HE+) or poor or no hemostatic efficacy (HE-). 
Table 1

Characteristics of the Patients at Baseline. ${ }^{*}$

\begin{tabular}{|c|c|c|}
\hline Characteristic & $\begin{array}{l}\text { Safety Population } \\
(\mathbf{N}=67)\end{array}$ & $\begin{array}{c}\text { Efficacy Population } \\
(\mathbf{N}=47)\end{array}$ \\
\hline Age - yr & $77.1 \pm 10.0$ & $77.1 \pm 10.1$ \\
\hline Male sex — no. $(\%)$ & $35(52)$ & $24(51)$ \\
\hline White race - no. $(\%)^{\dagger}$ & $54(81)$ & $36(77)$ \\
\hline Body-mass index ${ }^{*}$ & $28.1 \pm 6.3$ & $28.8 \pm 6.7$ \\
\hline Time from patient consent until andexanet bolus $-\mathrm{hr}$ & $1.7 \pm 0.8$ & $1.8 \pm 0.9$ \\
\hline Time from presentation until andexanet bolus $-\mathrm{hr}$ & $4.8 \pm 1.9$ & $4.8 \pm 1.8$ \\
\hline \multicolumn{3}{|l|}{ Estimated creatinine clearance - no. $(\%)$} \\
\hline$<30 \mathrm{ml} / \mathrm{min}$ & $6(9)$ & $4(9)$ \\
\hline 30 to $<60 \mathrm{ml} / \mathrm{min}$ & $31(46)$ & $25(53)$ \\
\hline$\checkmark 60 \mathrm{ml} / \mathrm{min}$ & $26(39)$ & $17(36)$ \\
\hline Missing data & $4(6)$ & $1(2)$ \\
\hline \multicolumn{3}{|l|}{ Indication for anticoagulation - no. $(\%)$} \\
\hline Atrial fibrillation & $47(70)$ & $32(68)$ \\
\hline Venous thromboembolism $\xi$ & $15(22)$ & $12(26)$ \\
\hline Atrial fibrillation and venous thromboembolism & $5(7)$ & $3(6)$ \\
\hline \multicolumn{3}{|l|}{ Medical history — no. (\%) } \\
\hline Myocardial infarction & $13(19)$ & $7(15)$ \\
\hline Stroke & $17(25)$ & $15(32)$ \\
\hline Deep-vein thrombosis & $20(30)$ & $16(34)$ \\
\hline Pulmonary embolism & $6(9)$ & $4(9)$ \\
\hline Atrial fibrillation & $49(73)$ & $34(72)$ \\
\hline Heart failure & $23(34)$ & $19(40)$ \\
\hline Diabetes mellitus & $23(34)$ & $17(36)$ \\
\hline \multicolumn{3}{|l|}{ Factor Xa inhibitor } \\
\hline \multicolumn{3}{|l|}{ Rivaroxaban } \\
\hline No. of patients & 32 & 26 \\
\hline Median daily dose $(\mathrm{IQR})-\mathrm{mg}$ & $20(15-20)$ & $20(20-20)$ \\
\hline Time from last dose to andexanet bolus - $\mathrm{hr}$ & $12.8 \pm 4.2$ & $12.0 \pm 4.1$ \\
\hline Baseline anti-factor Xa activity $-\mathrm{ng} / \mathrm{ml}$ & $247.4 \pm 186.0$ & $297.0 \pm 171.0$ \\
\hline Median unbound fraction of the plasma level (IQR) $-\mathrm{ng} / \mathrm{ml}$ & $16.7(10.2-25.5)$ & $19.3(12.0-26.9)$ \\
\hline \multicolumn{3}{|l|}{ Apixaban } \\
\hline No. of patients & 31 & 20 \\
\hline Median daily dose $(\mathrm{IQR}) — \mathrm{mg}$ & $5(5-10)$ & $5(5-10)$ \\
\hline Time from last dose to andexanet bolus - $\mathrm{hr}$ & $12.1 \pm 4.7$ & $11.0 \pm 4.7$ \\
\hline Baseline anti-factor Xa activity $-\mathrm{ng} / \mathrm{ml}$ & $137.7 \pm 102.3$ & $174.5 \pm 97.0$ \\
\hline
\end{tabular}




\begin{tabular}{|lcc|}
\hline Characteristic & $\begin{array}{c}\text { Safety Population } \\
(\mathbf{N}=\mathbf{6 7})\end{array}$ & $\begin{array}{c}\text { Efficacy Population } \\
\text { ( N = 47) }\end{array}$ \\
\hline Median unbound fraction of the plasma level (IQR) $-\mathrm{ng} / \mathrm{ml}$ & $9.4(6.0-19.2)$ & $10.5(8.1-19.2)$ \\
\hline Enoxaparin & & \\
\hline No. of patients & 4 & 1 \\
\hline Median daily dose (IQR) $-\mathrm{mg}$ & $90(80-150)$ & 200 \\
\hline Time from last dose to andexanet bolus $-\mathrm{hr}$ & $10.8 \pm 3.5$ & 13.1 \\
\hline Baseline anti-factor Xa activity $-\mathrm{IU} / \mathrm{ml}$ & $0.4 \pm 0.2$ & 0.6 \\
\hline
\end{tabular}

Plus-minus values are means \pm SD. Percentages may not total 100 because of rounding. IQR denotes interquartile range.

${ }^{\dagger}$ Race was reported by the investigators.

${ }^{*}$ The body-mass index is the weight in kilograms divided by the square of the height in meters.

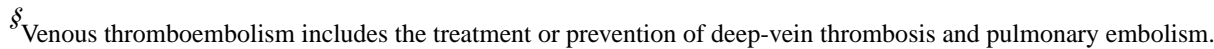


Table 2

Characteristics of Acute Major Bleeding Episodes and Clinical Outcomes. ${ }^{*}$

\begin{tabular}{|c|c|c|}
\hline Characteristic & $\begin{array}{l}\text { Safety Population } \\
(\mathbf{N}=67)\end{array}$ & $\begin{array}{c}\text { Efficacy Population } \\
(\mathbf{N}=47)\end{array}$ \\
\hline Gastrointestinal bleeding — no./total no. (\%) & $33 / 67(49)$ & $25 / 47(53)$ \\
\hline Patients receiving rivaroxaban & $20 / 33(61)$ & $16 / 25(64)$ \\
\hline Patients receiving apixaban & $11 / 33(33)$ & $8 / 25(32)$ \\
\hline \multicolumn{3}{|l|}{ Site of bleeding } \\
\hline Upper gastrointestinal tract & $9 / 33(27)$ & $7 / 25(28)$ \\
\hline Lower gastrointestinal tract & $10 / 33(30)$ & $8 / 25(32)$ \\
\hline Unknown & $14 / 33(42)$ & $10 / 25(40)$ \\
\hline Baseline hemoglobin $\unlhd 0 \mathrm{~g} / \mathrm{dl}$ & $20 / 33(61)$ & $16 / 25(64)$ \\
\hline Pretreatment red-cell transfusion & $21 / 33(64)$ & $19 / 25(76)$ \\
\hline Intracranial bleeding — no./total no. (\%) & $28 / 67(42)$ & 20/47 (43) \\
\hline Patients receiving rivaroxaban - no./total no. $(\%)$ & $10 / 28(36)$ & $8 / 20(40)$ \\
\hline Patients receiving apixaban - no./total no. (\%) & $17 / 28(61)$ & $12 / 20(60)$ \\
\hline Baseline score on Glasgow Coma Scale ${ }^{\dagger}$ & $14.1 \pm 1.7$ & $14.1 \pm 1.7$ \\
\hline Intracerebral site - no./total no. $(\%)$ & $14 / 28(50)$ & $12 / 20(60)$ \\
\hline Baseline score on modified Rankin scale ${ }^{t}$ & $3.0 \pm 1.8$ & $2.8 \pm 1.9$ \\
\hline \multicolumn{3}{|l|}{ Hematoma volume - no./total no. (\%) } \\
\hline$\unlhd 0 \mathrm{ml}$ & $8 / 14(57)$ & $8 / 12(67)$ \\
\hline 11 to $60 \mathrm{ml}$ & $6 / 14(43)$ & $4 / 12(33)$ \\
\hline Subdural site — no./total no. (\%) & $11 / 28(39)$ & $7 / 20(35)$ \\
\hline Baseline score on modified Rankin scale & $2.1 \pm 1.6$ & $1.4 \pm 1.5$ \\
\hline \multicolumn{3}{|l|}{ Maximal thickness - no./total no. (\%) } \\
\hline$\unlhd 0 \mathrm{~mm}$ & $8 / 11(73)$ & $5 / 7(71)$ \\
\hline$>10 \mathrm{~mm}$ & $3 / 11(27)$ & 2/7 (29) \\
\hline Subarachnoid site - no./total no. (\%) & $3 / 28(11)$ & $1 / 20(5)$ \\
\hline Other bleeding site - no./total no. (\%) & $6 / 67(9)$ & $2 / 47(4)$ \\
\hline Patients receiving rivaroxaban & $2 / 6(33)$ & $2 / 2(100)$ \\
\hline Patients receiving apixaban & $4 / 6(67)$ & 0 \\
\hline \multicolumn{3}{|l|}{ Site of bleeding } \\
\hline Nasal & $1 / 6(17)$ & 0 \\
\hline Pericardial, pleural, or retroperitoneal & $3 / 6(50)$ & $1 / 2(50)$ \\
\hline Genital or urinary & $1 / 6(17)$ & $1 / 2(50)$ \\
\hline Articular & $1 / 6(17)$ & 0 \\
\hline Pretreatment red-cell transfusion & $3 / 6(50)$ & $1 / 2(50)$ \\
\hline \multicolumn{3}{|l|}{ Clinical outcome - no./total no. (\%) } \\
\hline Death & $10 / 67(15)$ & $7 / 47(15)$ \\
\hline Thromboembolic event & $12 / 67(18)$ & $7 / 47(15)$ \\
\hline
\end{tabular}


* Plus-minus values are means $\pm \mathrm{SD}$.

${ }^{\dagger}$ Scores on the Glasgow Coma Scale range from 15 (normal) to 3 (deep coma).

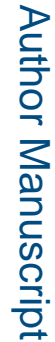

${ }^{*}$ Scores on the modified Rankin scale for global disability and handicap range from 0 (no symptoms or disability) to 6 (death). 